\title{
MẠTT QUASIGEOID - CƠ SỞ THỰC TẾ CỦA VIỆC XÂY DỰNG HÊ Độ CAO CHUẨN THEO LÝ THUYẾT CỦA MOLODENXKII M.X.
}

\author{
HÀ MINH HÒA \\ Viện Khoa học Đo đạc và Bản đồ
}

\section{Tóm tắt}

Bài báo khoa học này đã chứng minh ư điểm nổi bật của độ cao chuẩn được xác định trong trọng trưòng chuẩn của ellipsoid quy chiếu mà không đòi hỏi phải biết các quy luật phân bố mật độ vật chất ở lớp vỏ của Trái đất. Ngoài việc chưng minh được rằng mặt quasigeoid được xác định trong trọng truờng chuẩn của ellipsoid quy chiếu, không phải là mặt đẳng thế chuẩn, trùng với mặt geoid trên biển, trên co sở xây dụng được điều kiện Molodenxkii M.X. đối với mặt quasigeoid, bài báo này đã hệ thống các công thức được sủ dụng để khai thác các mô hình toàn cầu EGM, MDT để giải quyết các bài toán trắc địa vật lý trên phạm vi quốc gia hoặc khu vực.

\section{1. Đặt vấn đề}

Như đã trình bày trong tài liệu (Hà Minh Hòa, 2018), trong các tài liệu về Trắc địa vật lý trên thế giới đã nêu rõ rằng để giải quyết hai vấn đề của Stokes $\mathrm{G}$, khi giải quyết bài toán biên hỗn hợp, Molodenxkii M.X. đã đưa bài toán biên này vào trọng trường chuẩn của ellipsoid và đưa mặt biên - mặt telluroid - lên sát gần mặt vật lý Trái đất. Khi từ điểm $\mathrm{P}$ trên mặt vật lý Trái đất dựng đoạn thẳng $\mathrm{PQ}_{0}$ vuông góc với mặt ellipsoid và điểm $\mathrm{N}$ trên đoạn thẳng này nằm trên mặt telluroid,them vào đó điểm $\mathrm{Q}_{0}$ nằm trên mặt ellipsoid. thì đoạn $\mathrm{PN}$ bằng dị thường độ cao $\zeta_{P}$, đoạn $\mathrm{NQ}_{0}$ bằng độ cao chuẩn $H_{P}^{\gamma}$. Trong quá trình mô tả lý thuyết của Molodenxkii M.X., trong các tài liệu quốc tế về Trắc địa vật lý, ví dụ Jekeli C., 2000; Hofmann-Wellenhof B. and Moritz H., 2005; Ogorodova L.V., 2010, khi khẳng định độ cao chuẩn được xác định trong trọng trường chuẩn của ellipsoid, đã sử dụng mặt telluroid để định nghĩa độ cao chuẩn. Trong tài liệu (Pellinen, L.P., 1978) đã sử dụng định nghĩa của độ cao chuẩn dựa trên mặt telluroid để xây dựng lý thuyết chuyển các chênh cao đo từ trọng trường thực của Trái đất về trọng trường chuẩn của ellipsoid. Cách định nghĩa độ cao chuẩn dựa trên mặt telluroid làm cho khái niệm về độ cao chuẩn trở nên trừu tượng và không thực tế. Điều này đã dẫn đến sai lầm khi định nghĩa hệ độ cao Châu Âu EVRF2000. Ngoài ra, điều này còn gây nhầm lẫn khi coi mặt ellipsoid là "mặt khởi tính" của hệ độ cao chuẩn. Như đã chứng minh trong tài liệu (Hà Minh Hòa, 2014), mặt telluroid thực chất là hình ảnh của mặt vật lý trái đất mà theo đó mỗi điểm trên mặt vật lý trái đất được chiếu vào trọng trường chuẩn của ellipsoid quy chiếu theo phương vuông góc với mặt ellipsoid với khoảng cách chiếu bằng dị thường độ cao của điểm. Tuy nhiên, do các phương chiếu không song song với nhau và các giá trị dị thường độ cao tại các điểm trên mặt vật lý trái đất luôn khác nhau, nên hình ảnh của mặt telluroid không trùng với hình ảnh của mặt vật lý trái đất. Khi coi mặt ellipsoid là "mặt khởi tính" của hệ độ cao chuẩn, lý thuyết hệ độ cao chuẩn sẽ trái với lý thuyết hệ độ cao của Listing J.B., theo đó mặt khởi tính hệ độ cao phải là mặt geoid, và việc đo vẽ địa hình của mặt vật lý Trái đất được hiểu là đo vẽ mặt địa hình của mặt telluroid ?.

Bản thân Molodenxkii M.X. đã nhấn mạnh rằng khi xác định độ cao chuẩn chúng ta phải làm 
ngược lại: Trên đoạn thẳng $\mathrm{PQ}_{0}$ vuông góc với mặt ellipsoid từ điểm $\mathrm{P}$ dựng đoạn $\mathrm{PQ}$ bằng độ cao chuẩn quốc gia (cục bộ) $H_{P}^{\gamma}$. Qũy tích các điểm $\mathrm{Q}$ của các điểm $\mathrm{P}$ trên mặt vật lý Trái đất sẽ nằm trên mặt quasigeoid cục bộ. Do đoạn thẳng $\mathrm{PQ}_{0}$ bằng độ cao trắc địa $H_{P}$ của điểm $\mathrm{P}$, thêm vào đó $H_{P}=H_{P}^{\gamma}+\zeta_{P}$, nên đoạn thẳng $\mathrm{QQ}_{0}$ được gọi là độ cao quasigeoid quốc gia (cục bộ) và bằng dị thường độ cao quốc gia $\zeta_{P}$ của điểm $\mathrm{P}$ (xem Hình 1). Như vậy, độ cao chuẩn quốc gia $H_{P}^{\gamma}$ của điểm $\mathrm{P}$ trên mặt vật lý Trái đất đơn giản chỉ là khoảng cách từ điểm $\mathrm{P}$ đến mặt quasigeoid cục bộ theo đường vuông góc hạ từ điểm $\mathrm{P}$ đến mặt ellipsoid.

Như vậy, khi nghiên cứu lý thuyết của Molodenxkii M.X., chúng ta phải phân biệt hai mặt: Mặt telluroid được sử dụng để giải quyết bài toán biên hỗn hợp, còn mặt quasigeoid để xây dựng hệ độ cao chuẩn. Trong bài báo khoa học này, chúng ta sẽ tập trung nghiên cứu lý thuyết hệ độ cao chuẩn dựa trên mặt quasigeoid. Từ đây sẽ xác định được các đặc trưng cơ bản của mặt quasigeoid phục vụ việc giải quyết các bài toán trắc địa vật lý hiện đại. Trong các tài liệu về Trắc địa vật lý đều khẳng định mặt quasigeoid trùng với mặt geoid trên biển và các đại dương, mặt quasigeoid không phải là mặt đẳng thế trong trọng trường chuẩn của ellipsoid. Tuy nhiên những điều trên không được chứng minh. Trong bài báo khoa học này chúng ta sẽ chứng minh những điều này.

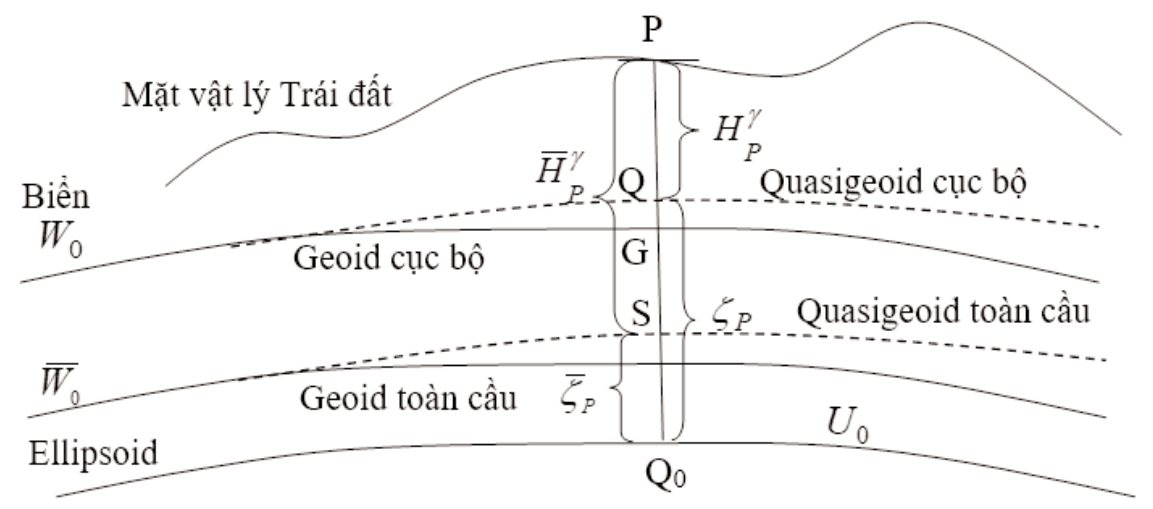

Hình 1: Mặt geoid và mặt quasigeoid

\section{Giải quyết vấn đề}

Trong các tài liệu (Hà Minh Hòa, 2010; Hà Minh Hòa, 2014) đã luận chứng cho khái niệm về mặt quasigeoid, theo đó "Mặt quasigeoid chi là hình ảnh của mặt geoid với thế trọng truờng thực $W=W_{0}$ trong trọng truờng chuẩn của ellipsoid và nhận được tù̀ phép ánh xạ mặt geoid này tù trọng truò̀ng thực của Trái đất về trọng trường chuẩn của ellipsoid theo đương vuông góc với mặt ellipsoid. Với một ellipsoid xác định, các geoid khác nhau sẽ có hình ảnh khác nhau trong trọng truờng chuẩn của ellipsoid này. Với một geoid đã xác định, nó có các hình ảnh khác nhau trong các trọng truoòng chuẩn khác nhau của các ellipsoid khác nhau”. Như vậy, mặt quasigeoid có mối quan hệ chặt chẽ với mặt geoid và được xác định phụ thuộc vào trọng trường chuẩn của ellipsoid cụ thể. Ngoài ra, mặt quasigeoid được xác định trong trọng trường chuẩn của ellipsoid.

Trong tài liệu Hà Minh Hòa, 2018 đã sử dụng công thức xác định giá trị trung bình của gia tốc lực trọng trường chuẩn $\overline{\gamma_{p}}$ tại điểm $\mathrm{P}$ nhu sau:

$$
\bar{\gamma}_{P}=\gamma_{0}-0,1543 \cdot H_{P}^{\gamma}+0,036 \cdot 10^{-6} \cdot\left(H_{P}^{\gamma}\right)^{2}
$$


ở đây $\gamma_{0}$ - gia tốc lực trọng trường chuẩn trên mặt ellipsoid.

Giá trị $\bar{\gamma}_{P}$ thực chất là giá trị trung bình của gia tốc lực trọng chuẩn giữa điểm $\mathrm{N}$ nằm trên telluroid và điểm $\mathrm{Q}_{0}$ nằm trên mặt telluroid. Giá trị trung bình trị (1) là giá trị cơ bản được sử dụng trong lý thuyết của Molodenxkii M.X..

Trong tài liệu (Hà Minh Hòa, 2016b) đã chứng minh công thức xác định độ cao chuẩn với việc sử dụng giá trị trung bình trị $\bar{F}_{\text {p }}$ (1) trong trường hợp sử dụng mặt quasigeoid làm mặt khởi tính cho hệ độ cao chuẩn. Với mục đích luận chứng chặt chẽ cho cơ sở khoa học của việc sử dụng mặt quasigeoid, không mất tính chất chung trong bài báo này chúng ta sẽ chứng minh rằng trong trường hợp sử dụng mặt quasigeoid cục bộ, độ cao trắc địa $H_{P}$, độ cao chuẩn quốc gia $H_{P}^{\gamma}$ và độ cao quasigeoid quốc gia $\zeta_{P}$ đều được tính toán nhờ giá trị trung bình trị $\bar{\gamma}_{P}(1)$. Trong trọng trường chuẩn của ellipsoid, các giá trị gia tốc lực trong trường chuẩn tại các điểm $\mathrm{P}, \mathrm{Q}$ được xác định theo các công thức sau:

$$
\begin{array}{cc}
\gamma_{P}=\gamma_{0}-0,3086 \cdot H_{P}+0,0723 \cdot 10^{-6} \cdot\left(H_{P}\right)^{2}, & <\mathrm{mGal}> \\
\gamma_{Q}=\gamma_{0}-0,3086 \cdot \zeta_{P}+0,0723 \cdot 10^{-6} \cdot\left(\zeta_{P}\right)^{2} & <\mathrm{mGal}>.
\end{array}
$$

Chúng ta sẽ nhận giá trị trung bình của gia tốc lực trọng trường chuẩn trên toàn cầu $\overline{\bar{\gamma}}=979764,5$ $m G a l$, độ cao quasigeoid lớn nhất $\mathfrak{\Xi}^{*}=100 \mathrm{~m}$, độ cao chuẩn $H^{\gamma}=8848 \mathrm{~m}$ của đỉnh Everest trên dãy Himalaya để tính toán.

Trong trọng trường chuẩn của ellipsoid, độ cao trắc địa $H_{P}$ được tính theo công thức:

$$
H_{P}=\frac{U_{0}-U_{P}}{\bar{\gamma}_{P}^{*}},
$$

ở đây $\bar{\gamma}_{P}^{*}$ là giá trị trung bình của gia tốc lực trọng trường chuẩn trên đoạn $\mathrm{PQ}_{0}$.

Lưu ý (2), giá trị $\bar{\gamma}_{P}^{*}$ được tính theo công thức:

$$
\bar{\gamma}_{P}^{*}=\frac{\gamma_{0}+\gamma_{P}}{2}=\gamma_{0}-0,1543 \cdot H_{P}+0,03615 \cdot 10^{-6} \cdot\left(H_{P}\right)^{2}<\mathrm{mGal}>.
$$

Khi thay $H_{P}=H_{P}^{\gamma}+\zeta_{P}$, vào (5) và lưu ý (1) chúng ta có quan hệ

$$
\frac{1}{\bar{\gamma}_{P}}-\frac{1}{\bar{\gamma}_{P}^{*}}=-\frac{0,1543 \cdot \zeta_{P}}{\bar{\gamma}_{P} \cdot \bar{\gamma}_{P}^{*}}+\frac{0,0723 \cdot 10^{-6} \cdot H_{P}^{\gamma} \cdot \zeta_{P}}{\bar{\gamma}_{P} \cdot \bar{\gamma}_{P}^{*}}+\frac{0,03615 \cdot 10^{-6} \cdot \zeta_{P}^{2}}{\bar{\gamma}_{P} \cdot \bar{\gamma}_{P}^{*}} \text {. }
$$

Với các giá trị $\bar{\gamma}_{P}=\bar{\gamma}_{P}^{*}=979764,5 \mathrm{mGal}, \zeta_{P}=100 \mathrm{~m}, H_{P}^{\gamma}=8848 \mathrm{~m}$ từ biểu thức trên suy ra hiệu $\frac{1}{\gamma_{p}} \frac{1}{\gamma_{P}^{*}}$ chỉ là đại lượng nhỏ ở mức $10^{-11}$. Do đó chúng ta hoàn toàn có cơ sở để nhận tỷ số $\frac{x}{\mathbb{F}_{*}^{*}}$ trong công thức (4) bằng $\frac{1}{\sqrt{*}}$ và khi sử dụng công thức (4) chúng ta tính giá trị trung bình $\mathscr{F}^{*}$ theo công thức (1) đối với giá trị trung bình $\overline{y_{x}}$

Tương tự, trong trọng trường chuẩn của ellipsoid độ cao chuẩn $H_{P}^{\gamma}$ được xác định theo công thức:

$$
H_{P}^{\gamma}=\frac{U_{Q}-U_{P}}{\bar{\gamma}_{P Q}},
$$

ở đây $\gamma_{\text {rag }}$ là giá trị trung bình của gia tốc lực trọng trường chuẩn trên đoạn $\mathrm{PQ}$. 


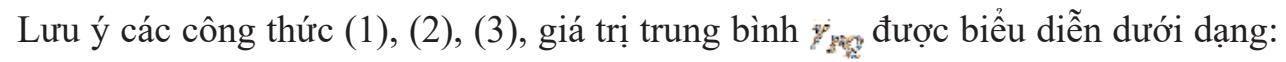

$$
\begin{aligned}
& \bar{\gamma}_{P Q}=\frac{\gamma_{P}+\gamma_{Q}}{2}=\gamma_{0}-0,1543 \cdot H_{P}^{\gamma}+0,03615 \cdot 10^{-6} \cdot\left(H_{P}^{\gamma}\right)^{2}-0,3086 \cdot \zeta_{P}+ \\
& +0,0723 \cdot 10^{-6} \cdot H_{P}^{\gamma} \cdot \zeta_{P}+0,0723 \cdot 10^{-6} \cdot \zeta_{P}^{2}=\bar{\gamma}_{P}-0,3086 \cdot \zeta_{P}+0,0723 \cdot 10^{-6} \cdot H_{P}^{\gamma} \cdot \zeta_{P}+ \\
& +0,0723 \cdot 10^{-6} \cdot \zeta_{P}^{2} .
\end{aligned}
$$

Từ biểu thức trên suy ra quan hệ:

$$
\frac{1}{\bar{\gamma}_{P}}-\frac{1}{\bar{\gamma}_{P Q}}=-\frac{0,3086 \cdot \zeta_{P}}{\bar{\gamma}_{P} \cdot \bar{\gamma}_{P Q}}+\frac{0,0723 \cdot 10^{-6} \cdot H_{P}^{\gamma} \cdot \zeta_{P}}{\bar{\gamma}_{P} \cdot \bar{\gamma}_{P Q}}+\frac{0,0723 \cdot 10^{-6} \cdot \zeta_{P}^{2}}{\bar{\gamma}_{P} \cdot \bar{\gamma}_{P Q}} .
$$

Với các giá trị $\quad \bar{\gamma}_{P}=\bar{\gamma}_{P Q}=979764,5 \mathrm{mGal}, \zeta_{P}=100 \mathrm{~m}, H_{P}^{\gamma}=8848 \mathrm{~m}$ từ biểu thức trên suy ra hiệu

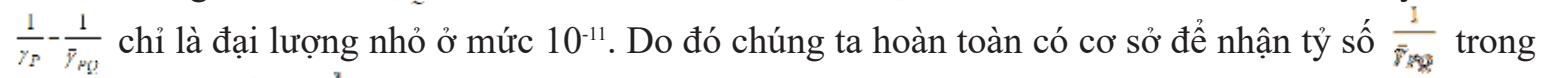
công thức (6) bằng $\frac{1}{\bar{P}_{\infty}}$ và khi sử dụng công thức (6) chúng ta tính giá trị trung bình $\bar{F}_{F_{g}}$ theo công thức (1) đối với giá trị trung bình $\bar{y}_{x_{x}}$

Cuối cùng, trong trọng trường chuẩn của ellipsoid, độ cao quasigeoid $\zeta_{P}$ được biểu diễn dưới dạng:

$$
\zeta_{P}=\frac{U_{0}-U_{Q}}{\bar{\gamma}_{Q_{0} Q}},
$$

ở đây $\bar{\gamma}_{Q_{0} Q}$ là giá trị trung bình của gia tốc lực trọng trường chuẩn trên đoạn $\mathrm{Q}_{0} \mathrm{Q} ; U_{0}$ - thế trọng trường chuẩn trên mặt ellipsoid.

Từ công thức (3) chúng ta suy ra công thức xác định giá trị trung bình $\bar{\gamma}_{Q_{0} Q}$ ở dạng sau:

$$
\bar{\gamma}_{Q_{0} Q}=\frac{\gamma_{Q}+\gamma_{0}}{2}=\gamma_{0}-0,1543 \zeta_{P}+0,0361510^{-6} \cdot\left(\zeta_{P}\right)^{2} .
$$

Lưu ý (10) từ biểu thức trên chúng ta nhận được quan hệ:

$$
\frac{1}{\bar{\gamma}_{Q_{0} Q}}-\frac{1}{\bar{\gamma}_{P}}=-\frac{0,1543 \cdot H_{P}^{\gamma}}{\bar{\gamma}_{Q_{0} Q} \cdot \bar{\gamma}_{P}}+\frac{0,036 \cdot 10^{-6} \cdot\left(H_{P}^{\gamma}\right)^{2}}{\bar{\gamma}_{Q_{0} Q} \cdot \bar{\gamma}_{P}}+\frac{0,1543 \cdot \zeta_{P}}{\bar{\gamma}_{Q_{0} Q} \cdot \bar{\gamma}_{P}}-\frac{0,03615 \cdot 10^{-6} \cdot \zeta_{P}^{2}}{\bar{\gamma}_{Q_{0} Q} \cdot \bar{\gamma}_{P}} .
$$

Với các giá trị $\bar{\gamma}_{P}=\bar{\gamma}_{Q_{0} Q}=979764,5 \mathrm{mGal}, \zeta_{P}=100 \mathrm{~m}, H_{P}^{\gamma}=8848 \mathrm{~m}$ từ biểu thức trên suy ra hiệu $\bar{\gamma}_{T}-\gamma_{Q_{1} Q}$ chỉ là đại lượng nhỏ ở mức $10^{-9}$. Như vậy chúng ta hoàn toàn có cơ sở để tỷ số $\frac{1}{\bar{\gamma}_{Q_{0}} Q}$ trong công thức (7) bằng $\frac{i}{\bar{\gamma}_{s}}$ và khi sử dụng công thức (7) chúng ta tính giá trị trung bình $\bar{\gamma}_{Q_{0} Q}$ theo công thức (1) đối với giá trị trung bình $\bar{\gamma}_{P}$.

Như vậy chúng ta đã chứng minh một vấn đề quan trọng: Khi sử dụng mặt telluroid hay mặt quasigeoid, trong lý thuyết của Molodenxkii M.X. giá trị trung bình của gia tốc lực trọng trường chuẩn luôn được tính theo công thức (1) và được sử dụng để tính toán độ cao trắc địa $H_{P}$, độ cao chuẩn $H_{P}^{\gamma}$ và độ cao quasigeoid $\zeta_{P}$ Chính điều này là sự đảm bảo mối liên kết giữa việc tính toán độ cao chuẩn và dị thường độ cao được tính toán theo các công thức $(1),(2)$ dựa trên mặt telluroid trong tài liệu (Hà Minh Hòa, 2018) với độ cao chuẩn và độ cao quasigeoid được tính toán theo các công thức (6), (7) ở trên khi sử dụng mặt quasigeoid. Không những thế, khi sử dụng mặt telluroid hay mặt quasi- 
geoid, điều nêu trên luôn đảm bảo quan hệ: Độ cao trắc địa $H_{P}$ bằng tổng của độ cao chuẩn $H_{P}^{\gamma}$ và độ cao quasigeoid $\zeta_{P}$.

Bây giờ chúng ta sẽ chứng minh rằng mặt quasigeoid trùng với mặt geoid trên biển và các đại dương, thêm vào đó mặt quasigeoid không phải là mặt đẳng thế chuẩn trong trọng trường chuẩn của ellipsoid. Mối quan hệ giữa độ cao chính $H_{P}^{g}$ và độ cao chuẩn $H_{P}^{\gamma}$ của điểm $\mathrm{P}$ được biểu diễn qua công thức đã biết (Pellinen, L.P., 1978; Hofmann-Wellenhof B. and Moritz H., 2005):

$$
W_{0}-W_{P}=\bar{g}_{P} \cdot H_{P}^{g}=\bar{\gamma}_{P} \cdot H_{P}^{\gamma},
$$

ở đây $\bar{g}_{P}$ - giá trị trung bình tích phân của gia tốc lực trọng trường thực tại điểm $\mathrm{P} ; W_{0}$ - thế trọng trường thực trên mặt geoid; $W_{P}$ - thế trọng trường thực tại điểm $\mathrm{P}$ trên mặt vật lý Trái đất..

Từ quan hệ (8) suy ra hai công thức:

$$
\begin{gathered}
H_{P}^{\gamma}=H_{P}^{g}+\frac{\bar{g}_{P}-\bar{\gamma}_{P}}{\bar{\gamma}_{P}} \cdot H_{P}^{g}, \\
d H=H_{P}^{\gamma}-H_{P}^{g}=\frac{\bar{g}_{P}-\bar{\gamma}_{P}}{\bar{\gamma}_{P}} \cdot H_{P}^{g} .
\end{gathered}
$$

Từ công thức (9) chúng ta thấy rằng sự khác nhau giữa độ cao chuẩn $H_{P}^{\gamma}$ và độ cao chính $H_{P}^{g}$ được gây ra do sự không trùng nhau của trọng trường chuẩn của ellipsoid và trọng trường thực của Trái đất. Công thức (9) mô tả phép ánh xạ độ cao chính từ trọng trường thực của Trái đất đi một đoạn $\mathrm{dH}$ (10) theo đường vuông góc $\mathrm{PQ}_{0}$ với mặt ellipsoid thành độ cao chuẩn trong trọng trường chuẩn của ellipsoid. Từ quan hệ đã biết:

$$
H_{P}=H_{P}^{g}+N_{P}=H_{P}^{\gamma}+\zeta_{P},
$$

và công thức (9) chúng ta có công thức:

$$
\zeta_{P}=N_{P}-\frac{\bar{g}_{P}-\bar{\gamma}_{P}}{\bar{\gamma}_{P}} \cdot H_{P}^{g} .
$$

Công thức trên biểu diễn phép ánh xạ độ cao geoid $N_{P}$ từ trọng trường thực của Trái đất theo đường vuông góc $\mathrm{PQ}_{0}$ với mặt ellipsoid thành độ cao quasigeoid $\zeta_{P}$ trong trọng trường chuẩn của ellipsoid. Bây giờ đặt biểu thức $H_{P}^{\gamma}=H_{P}-\zeta_{P}$ vào công thức $(8)$ chúng ta nhận được biểu thức:

$$
\bar{\gamma}_{P} \cdot \zeta_{P}=-W_{0}+W_{P}+\bar{\gamma}_{P} \cdot H_{P} .
$$

Thay độ cao trắc địa $H_{P}$ từ (4) vào biểu thức trên và lưu ý thế nhiễu $T_{P}=W_{P}-U_{P}$ tại điểm $\mathrm{P}$ trên mặt vật lý Trái đất, biểu thức trên có dạng:

$$
\bar{\gamma}_{P} \cdot \zeta_{P}=-W_{0}+W_{P}+U_{0}-U_{P}=-W_{0}+U_{0}+T_{P},
$$

Mặt khác từ công thức (7) suy ra:

$$
U_{Q}=U_{0}-\bar{\gamma}_{P} \cdot \zeta_{P} .
$$

Từ (11), (12) chúng ta nhận được quan hệ (Hà Minh Hòa, 2010; Hà Minh Hòa, 2014):

$$
U_{Q}=W_{0}-T_{P}
$$

Sự tồn tại thế nhiễu $T_{P}$ khác 0 tại điểm $\mathrm{P}$ trên mặt vật lý Trái đất là do sự không trùng nhau giữa 
trọng trường thực của Trái đất và trọng trường chuẩn của ellipsoid. Điều này cho phép lý giải sự khác nhau giữa thế trọng trường chuẩn $U_{Q}$ của điểm $\mathrm{Q}$ trên mặt quasigeoid và thế trọng trường $W_{0}$ của mặt geoid khi dựa trên quan hệ (13). Ngoài ra, công thức (13) cho thấy rằng do các giá trị thế nhiễu trên tập hợp các điểm $\mathrm{P}$ trên mặt vật lý Trái đất luôn khác nhau, nên các giá trị của các thế trọng trường chuẩn $U_{Q}$ của tập hợp các điểm $\mathrm{Q}$ tương ứng trên mặt quasigeoid luôn khác nhau. Điều này có nghĩa là mặt quasigeoid không phải là mặt đẳng thế chuẩn trong trọng trường chuẩn của ellipsoid.

Bây giờ thay $U_{Q}(13)$ vào công thức (7), chúng ta lại nhận được công thức Bruns:

$$
\zeta_{P}=\frac{T_{P}}{\bar{\gamma}_{P}}+\frac{U_{0}-W_{0}}{\bar{\gamma}_{P}} .
$$

Từ công thức (13) chúng ta suy ra điều kiện:

$$
C_{P}=W_{0}-W_{P}=U_{Q}-U_{P}
$$

Trong tài liệu (Hà Minh Hòa, 2017a) đã gọi điều kiện (14) là điều kiện Molodenxkii M.X. đối với mặt quasigeoid cục bộ. Như vậy, từ định nghĩa độ cao chuẩn trong công thức (8), chúng ta suy ra điều kiện (14) đối với mặt quasigeoid. Khi sử dụng mặt quasigeoid làm mặt khởi tính cho hệ độ cao chuẩn, quan hệ (13) và điều kiện (14) cho phép giải quyết nhiều vấn đề lý thuyết và thực tế của lý thuyết Molodenxkii M.X.. Từ điều kiện (14) chúng ta suy ra công thức xác định độ cao chuẩn $H_{P}^{\gamma}$ tương ứng với mặt quasigeoid ở dạng sau:

$$
H_{P}^{\gamma}=\frac{C_{P}}{\bar{\gamma}_{P}}=\frac{W_{0}-W_{P}}{\bar{\gamma}_{P}}=\frac{U_{Q}-U_{P}}{\bar{\gamma}_{P}} .
$$

Công thức (15) được xây dựng khi chọn mặt quasigeoid làm mặt khởi tính cho hệ độ cao chuẩn. Trong khi đó, như đã trình bày trong tài liệu (Hà Minh Hòa, 2018), trong trường hợp sử dụng mặt telluroid để mô tả độ cao chuẩn, chúng ta không thể làm rõ mặt khởi tính của hệ độ cao chuẩn là mặt nào.

Khi nhận giá trị trung bình tích phân của gia tốc lực trọng trường thực $\bar{g}$ trên toàn cầu bằng $9,80665 \mathrm{~m}^{2} \cdot \mathrm{s}^{-2}$ (The International System of Units (SI)), còn giá trị trung bình của gia tốc lực trọng trường chuẩn $\bar{F}$ trên toàn cầu bằng $9,797645 \mathrm{~m}^{2} \cdot \mathrm{s}^{-2}$ (Lemoine F.G., Kenyon S.C., Factor J.K., Trimmer R.G., Pavlis N.K., et al., 1998), chúng ta có đại lượng:

$$
\frac{\bar{g}-\bar{\gamma}}{\bar{\gamma}}=9,191 \cdot 10^{-4} \text {. }
$$

Trên biển và các đại dương, giá trị độ cao chính $H^{g}$ của mặt địa hình biển chỉ ở mức $\pm 2 \mathrm{~m}$ (Pellinen L.P., 1978; Rapp R.H.; Balasubramania N., 1992). Thay đại lượng (16) và $H_{P}^{g}= \pm 2 m$ vào công thức $(10)$, chúng ta nhận được giá trị $d H= \pm 1,8 \mathrm{~mm}$. Điều này có nghĩa là trên các biển và các đại dương, độ chênh giữa độ cao chuẩn và độ cao chính nhỏ bỏ qua, tức mặt quasigeoid được coi là trùng với mặt geoid. Với ý nghĩa này, mặt quasigeoid có đầy đủ tư cách là mặt khởi tính của hệ độ cao chuẩn theo định nghĩa về hệ độ cao của Listing J.B.. Tuy vậy, chúng ta phải phân biệt rõ ràng rằng mặt geoid là mặt đẳng thế trong trọng trường thực của Trái đất, còn mặt quasigeoid mặc dù trùng với mặt geoid trên các biển và các đại dương thế giới, nhưng nó không phải là mặt đẳng thế trong trọng trường thực của Trái đất bởi vì nó chỉ được xác định trong trọng trường chuẩn của ellipsoid, thêm vào đó nó cũng không phải là mặt đẳng thế chuẩn trong trọng trường chuẩn này. 
Với việc sử dụng mặt quasigeoid là mặt khởi tính của hệ độ cao chuẩn, các kết quả đo đạc địa hình chi tiết sẽ cho phép xây dựng các bản đồ địa hình các tỷ lệ khác nhau mô tả địa hình của bề mặt trái đất tương ứng với mặt quasigeoid. Trong khi đó việc sử dụng mặt ellipsoid làm mặt khởi tính hệ độ cao chuẩn cho phép mô tả mặt telluroid, chứ không phải địa hình của bề mặt trái đất. Chúng ta cũng cần thiết phải nhấn mạnh thêm rằng như đã trình bày ở trang 365 của tài liệu Hà Minh Hòa, 2014, mặt telluroid là hình chiếu của mặt vật lý trái đất vào trọng trường chuẩn của ellipsoid theo phương vuông góc với mặt ellipsoid đi một khoảng cách bằng dị thường độ cao của điểm trên mặt vật lý trái đất. Do các điểm trên mặt vật lý trái đất có các dị thường độ cao khác nhau, nên mặt telluroid không giống mặt vật lý trái đất

Việc sử dụng mặt quasigeoid cho phép giải quyết nhiều vấn đề lý thuyết và thực tế liên quan đến việc khai thác các mô hình toàn cầu như mô hình trọng trường trái đất EGM, mô hình Địa hình mặt biển trung bình toàn cầu $\mathrm{V} . . \mathrm{v}$ để giải quyết các bài toán trắc địa vật lý trong phạm vi quốc gia và khu vực. Dựa trên quan hệ (14), trong (Hà Minh Hòa, 2014) đã chứng minh các công thức chuyển đổi độ cao chuẩn, độ cao quasigeoid giữa các hệ triều. Đối với điểm $\mathrm{P}$ trên mặt vật lý trái đất, khi ký hiệu $\bar{H}_{P}^{\gamma}$ là độ cao chuẩn toàn cầu và $\bar{\zeta}_{P}$ là độ cao quasigeoid toàn cầu tương ứng với mặt quasigeoid toàn cầu (xem Hình 1$), H_{P}^{\gamma}$ là độ cao chuẩn cục bộ và $\zeta_{P}$ là độ cao quasigeoid cục bộ tương ứng với mặt quasigeoid cục bộ, thêm vào đó mặt quasigeoid toàn cầu tương ứng với mặt geoid toàn cầu với thế trọng trường $\overline{W_{0}}$ còn mặt quasigeoid cục bộ tương ứng với mặt geoid cục bộ với thế trọng trường $W_{0}$ được xác định tại trạm nghiệm triều 0 của quốc gia hoặc khu vực, tương tự như quan hệ (14) đối với mặt quasigeoid cục bộ, đối với mặt quasigeoid toàn cầu chúng ta có quan hệ:

$$
\bar{W}_{0}-W_{P}=U_{S}-U_{P},
$$

ở đây $\mathrm{S}$ là điểm cắt của đường vuông góc $\mathrm{PQ}_{0}$ với mặt quasigeoid toàn cầu.

Dựa trên các quan hệ (14), (17) chúng ta sẽ có các công thức sau:

$$
\begin{gathered}
\zeta_{P}=\bar{\zeta}_{P}+\frac{\bar{W}_{0}-W_{0}}{\bar{\gamma}_{P}}, \\
\bar{H}_{P}^{\gamma}=H_{P}^{\gamma}+\frac{\bar{W}_{0}-W_{0}}{\bar{\gamma}_{P}} .
\end{gathered}
$$

Các công thức nêu trên đã được chứng minh trong các tài liệu (Hà Minh Hòa, 2007; Hà Minh Hòa, 2014) theo các phương pháp khác nhau và đã được sử dụng trong tài liệu (Hà Minh Hòa, và nnk, 2012) để xác định thế trọng trường $W_{0}=62636847,2911 \mathrm{~m}^{2} \cdot \mathrm{s}^{-2}$ của mặt geoid cục bộ Hòn Dấu, sát nhất với mặt biển trung bình nhiều năm tại trạm nghiệm triều Hòn Dấu (trạm nghiệm triều 0 của Việt $\mathrm{Nam}$ ), dựa trên geoid toàn cầu với thế trọng trường $\bar{W}_{0}=626368560 \mathrm{~m}^{2} \cdot \mathrm{s}^{-2}$ (Petit G., Luzum B., 2010).

Từ các quan hệ (14) và (17) chúng ta nhận được đại lượng:

$$
H_{0}=\frac{\bar{W}_{0}-W_{0}}{\bar{\gamma}_{P}}=\frac{U_{S}-U_{Q}}{\bar{\gamma}_{P}}
$$

là độ cao của mặt quasigeoid cục bộ so với mặt geoid toàn cầu.

Do đã xác định được các giá trị thế trọng trường $W_{0}$ và $\overline{W_{0}}$ của mặt geoid cục bộ được xác định tại trạm nghiệm triều Hòn Dấu và mặt geoid toàn cầu, nên đối với hệ độ cao Hải Phòng 1972 chúng ta nhận được $H_{0}=0,890$ m (Hà Minh Hòa, và nnk, 2012). Trong tài liệu (Hà Minh Hòa, 2016a) đã 
chứng minh sự song song của mặt quasigeoid cục bộ Hòn Dấu với mặt quasigeoid toàn cầu trên phạm vi toàn cầu. Đây là cơ sở khoa học của việc sử dụng công thức (18) để chuyển độ cao quasigeoid toàn cầu thành độ cao quasigeoid hỗn hợp cục bộ phục vụ việc xây dựng mô hình quasigeoid hỗn hợp quốc gia và của việc sử dụng công thức (19) để chuyển đổi độ cao địa hình biển toàn cầu thành độ cao địa hình biển cục bộ phục vụ việc xây dựng mô hình Địa hình mặt biển trung bình trên vùng biển Việt Nam.

Với mục đích thống nhất các hệ độ cao của các nước khác nhau trong hệ độ cao thống nhất trên cơ sở sử dụng mặt geoid đã biết thế trọng trường $W_{0}$ tại trạm nghiệm triều 0 của khu vực, trên thế giới đã sử dụng phương pháp bình sai theo các hệ địa thế thay cho các chênh cao đo, ví dụ hệ độ cao Châu Âu EVRF2007 (Ihde J., 2007). Ở Việt Nam với việc xác định được thế trọng trường $W_{0}=$ $62636847,2911 \mathrm{~m}^{2} . \mathrm{s}^{-2}$ của mặt geoid cục bộ Hòn Dấu trong tài liệu (Hà Minh Hòa, Nguyễn Thị Thanh Hương, 2015a) đã tiến hành thử nghiệm bình sai theo các hiệu địa thế đối với mạng lưới độ cao hạng I, II miền Bắc. Trong trường hợp trên các điểm độ cao hạng I, II có đo GNSS và xử lý dữ liệu GNSS trong ITRF, các giá trị thế trọng trường của các mốc độ cao hạng I, II có thể được sử dụng để hiệu chỉnh các hệ số điều hòa của mô hình trọng trường trái đất EGM (Hà Minh Hòa, 2015b) hoặc để xây dựng mô hình Địa thế quốc gia (Hà Minh Hòa, 2017b).

\section{Kết luận}

Khi sử dụng lý thuyết của Molodenxkii M.X., chúng ta phải phân biệt hai mặt telluroid và quasigeoid, theo đó mặt telluroid được dựng lên nhờ các độ cao chuẩn của các điểm nằm trên mặt telluroid và được sử dụng làm mặt biên của bài toán biên hỗn hợp, tuy nhiên mặt quasigeoid trùng với mặt geoid trên biển được sử dụng để làm mặt khởi tính cho hệ độ cao chuẩn. Điều này làm cho hệ độ cao chuẩn hoàn toàn phù hợp với lý thuyết hệ độ cao của Listing J.. Việc các độ cao chuẩn của các điểm trên mặt vật lý trái đất được xác định trong trọng trường chuẩn của ellipsoid cho phép xác định chúng một cách đơn trị mà không cần biết quy luật phân bố mật độ vật chất trong lớp vỏ của Trái đất. Đây là ưu điểm nổi bật của độ cao chuẩn so với độ cao chính. Trên cơ sở tổng hợp các kết quả nghiên cứu đã được công bố từ trước của tác giả bài báo khoa học này, việc đề xuất điều kiện Molodenxkii M.X. đối với mặt quasigeoid cục bộ dưới dạng công thức (14) cho phép chứng minh mặt quasigeoid được xác định trong trọng trường chuẩn của ellipsoid quy chiếu, không phải là mặt đẳng thế chuẩn, trùng với mặt geoid trên biển và xây dựng được các công thức (18), (19), (20) phục vụ việc khai thác các mô hình toàn cầu EGM, MDT để giải quyết các bài toán trắc địa vật lý trong phạm vi quốc gia và khu vực. Để kết thúc bài báo khoa học này, mượn lời khẳng định của Sjoberg L.E. trong công trình (Sjoberg, L.E., 2013) rằng hệ độ cao chuẩn là triển vọng tương lai xây dựng hệ độ cao ở các nước ./.O

\section{Tài liệu tham khảo}

[1]. Hà Minh Hòa, 2007. Giải quyết một số vấn đề liên quan đến việc chuyển hệ độ cao được xác định từ mặt nước biển trung bình ở trạm thủy triều về mặt quasigeoid toàn cầu. Tạp chi Địa chính, số 2, tháng 4/2007, trg. 3 - 11, ISSN: 0866- 7705.

[2]. Hà Minh Hòa, 2010. Tiếp cận khái niệm về mặt Quasigeoid. Tạp chí Khoa học Đo đạc và Bản đồ, No3, 3/2010, trg. 3 - 15. ISSN: 0866- 7705.

[3]. Hà Minh Hòa, và nnk, 2012. Nghiên cứu cơ sở khoa học của việc hoàn thiện hệ độ cao gắn liền với việc xây dựng hệ tọa độ động lực quốc gia, 247 trg.. Đề tài khoa học và công nghệ cấp Bộ Tài nguyên và Môi trưòng giai đoạn 2010 - 2012. Hà Nội - 2012. 
[4]. Hà Minh Hòa, 2014. Lý thuyết và thực tiễn của Trọng lực trắc địa. Nhà Xuất bản Khoa học và Kỹ thuật, 592 trg., Hà Nội - 2014. ISBN: 978-604-67- 0301-3.

[5]. Hà Minh Hòa, Nguyễn Thị Thanh Hương, 2015a. Nghiên cứu hoàn thiện phương pháp xử lý toán học mạng lưới độ cao hạng I, II quốc gia trong hệ độ cao hiện đại ở Việt Nam. Đề tài nghiên cứu khoa học và công nghệ cấp Bộ Tài nguyên và Môi trường giai đoạn 2012 - 2015, Hà Nội - 2015.

[6]. Ха Минь Хоа, 2015b. Развитие государственной высотной системы Вьетнама на основе использования локальной модели геоида. Известия вузов Геодезия и Аэрофотосьёька, №2, c. 10 - 13, ISSN 0536-101X, Московский Университет Геодезии и Картографии, Россия.

Hà Minh Hòa, 2015b. Phát triển hệ độ cao quốc gia Việt Nam dựa trên mô hình geoid cục bộ. Izv. Vuzov. Geodezia I Aerophotoxemka, No2, trg. 10-13, Trường Đại học Tổng hợp Trắc địa và Bản đồ Matxcơva, Liên bang Nga. (TiếngNga).

[7]. Hà Minh Hòa, 2016a. Nghiên cứu sự thay đổi độ cao giữa mặt quasigeoid cục bộ Hòn Dấu và mặt quasigeoid toàn cầu trên phạm vi toàn cầu. Tạp chi Khoa hoc Đo đạc và Bản đồ, số 28 , tháng 06/2016, trg. 1 - 7, ISSN: 0866- 7705.

Hà Minh Hòa, 2016b. Nghiên cứu các đặc trưng cơ bản của mặt quasigeoid. Tạp chi Khoa hoc Đo đạc và Bản đồ, số 29, tháng 09/2016, trg. 1 - 9, ISSN: 0866- 7705.

[8]. Ха Минь Хоа, 2017а. Изучение некоторых специфических характеристик квазигеоида для решения задач физической геодезии на современной эпохе. Международный научно исследовательский журнал “Успехи современной науки и образования", Том 4 No. 1, 01/2017, c. 208 - 216. ВАК РФ, РИНЦ (eLibrary.ru), ERIH PLUS, AGRIS, ISSN 2412 - 9631, Типография “Эпицентр”, http://www.modernsciencejournal.org/.

Hà Minh Hòa, 2017a. Nghiên cứu một số các đặc trưng đặc thù của mặt quasigeoid để giải quyết các bài toán trắc địa vật lý trong kỷ nguyên hiện đại. Tạp chí Nghiên cưu khoa học quốc tế "Các thành tưu Khoa học hiện đại và Giáo duc", Tập 4, No.1, 01/2017, trg. 208 - 216, VAK RF (Liên bang Nga), RINTX (eLibrary.ru), ERIH PLUS, AGRIS, ISSN 2412 - 9631, Nhà in "Epicentr". http://modernsciencejournal.org.

[9]. Hà Minh Hòa, 2017b. Sử dụng phương pháp collocation để xác định thế trọng trường trên điểm GNSS dựa trên các thế trọng trường của các điểm độ cao hạng I, II ổn định và mô hình EGM2008. Kỷ yếu Hội nghị Khoa học \& Công nghệ lần thứ 15 ngày 20 tháng 10 năm 2017, Phân ban Địa tin học, trg. 1- 13. ISBN: 978 - 604 - 73 - 5642 - 3. Trường Đại học Bách khoa Tp. Hồ Chí Minh.

[10]. Hà Minh Hòa, 2018. Các ứng dụng rộng rãi của lý thuyết Molodenxkii M.X. trong Trắc địa vật lý trên thế giới. Tạp chí Khoa học Đo đạc và Bản đồ, số 37, tháng 06/2018, trg. 1 - , ISSN: 08667705 .

[11]. Hofmann-Wellenhof B. and Moritz H., 2005. Physical Geodesy, 2nd edition, Springer, Wien - New York

[12]. Ihde, J., 2007. Final Report of IIG ICP 1.2 for the period 2003 - 2007. IAG Inter Commission Project, ICO 1.2 Vertical Reference Frames. 30 p.

Jekeli, C., 2000. Heights, the geopotential and vertical datums. Report No. 459. The Ohio State University, Columbus, $35 \mathrm{p}$. 
[13]. Lemoine, F.G., Kenyon, S.C., Factor, J.K., Trimmer, R.G., Pavlis, N.K., et al, 1998. The Development of the Joint NASA GSFC and NIMA Geopotential Model EGM96, NASA Goddard Space Flight Center, NASA Technical Report NASA/TP-1996\8-206861 Greenbelt, Maryland, 20771 USA, July 1998).

[14]. Огородова, Л. В., 2010. Нормальное поле и определение аномального потенциала. Москва, МИИГАиК, 105 с.,ISBN 978-5-91188-025-5.

Ogorodova, L.V., 2010.Trường chuẩn và xác định thế dị thường. Matxcơva, MIIGAiK, 105 trg., ISBN 978-5-91188-025-5.

[15]. Пеллинен, Л. П., 1978. Высшая геодезия (теоретичесрая геодезия). Москва. Недра, $264 \mathrm{c}$.

Pellinen, L.P., 1978. Trắc địa cao cấp (trắc địa lý thuyết). Matxcơva, Nedra, 264 trg. (TiếngNga).

[16]. Petit, G., Luzum, B., 2010. IERS Conventions (2010). IERS Technical Note No 36, Verlag dés Bundesamts fur Kartographie und Geodasie. Frankfurt am Main 2010, 179 pp.

Rapp, R.H.; Balasubramania, N., 1992. A conceptual formulation of a World height system. Dept. Geod. Sci. Rep. N421. Ohio State University, Columbus, Ohio.

[17]. Sjoberg, L.E., 2013. The geoid or quasigeoid - which reference surface should be preferred for a national height system ?. Journal of Geodetic Science, 3 (2), 103-109, doi: 10.2478/jors-20140013

[18]. The International System of Units (SI). National Institute of Standards and Technology (NIST), U.S. Department of Commerce, INST Special Publication 330, 2008 Edition. Ed. Taylor B.N. and Thompson A., 97 p.O

\section{Summary} theory

A quasigeoid - practical base of construction of a normal height system by Molodenxkii's

\section{Ha Minh Hoa}

Vietnam Institute of Geodesy and Cartography

This scientific article proved a remarkable advantage of a normal height determined in a normal gravitational field of reference ellipsoid without law of mass distribution in the Earth's crust. Apart from provement that the quasigeoid has been determined in the normal gravitational field of reference ellipsoid, isn't the normal equipotential surface, is coincident with the geoid in the seas, on base of construction of Molodenxkii's condition for the quasigeoid, this article systematized formulas used for exploitation of the global models EGM, MDT in solving of tasks of the physical geodesy in domains of country or region. $\bigcirc$ 